\title{
Biotechnology for bacteriocin synthesis using photostimulation
}

\author{
Anna $V$. Diachkova ${ }^{1,2, *}$, Anna A. Nogina ${ }^{1}$, Sergey L. Tikhonov ${ }^{1}$, Natalia $V$. Tikhonova ${ }^{1}$, \\ Dina $G$. Popova ${ }^{3}$, and Leonid $S$. Kudryashov ${ }^{3}$ \\ ${ }^{1}$ Ural State University of Economics, Department of food engineering, 620144 Ekaterinburg, Russia \\ ${ }^{2}$ Ural Federal University named after the first President of Russia B. N. Eltsin, Department of \\ economic theory and economic policy, 620002 Ekaterinburg, Russia \\ ${ }^{3}$ Kemerovo State University, Department of Management of Quality, 650010 Kemerovo, Russia, \\ ${ }^{4}$ V.M. Gorbatov Federal Research Center for Food Systems of Russian Academy of Sciences, \\ 109316 Moscow, Russia
}

\begin{abstract}
This article presents results of a study of the effect of blue color on the bacteriocin activity. It was revealed that photostimulation of Lactobacillus Lactis with light in the blue spectrum $(435-470 \mathrm{~nm})$ with a light flux intensity of 1800 mcd for 50-60 minutes positively affects the bacteriocin activity (increases by $60.1 \%$ ), while titer of the Lactobacillus lactis is 2 times higher. It was found that it was in the first 18 hours that the irradiated samples had a greater difference in terms of activity and titers than unirradiated samples. Oxidative stress affects the culture's viability, both in samples treated with blue light and in the untreated cultures. However, impact intensity on the treated cultures is weaker. These outcomes indicate the possibility of using blue light for stimulating bacteriocin biosynthesis in order to use it as a food additive for prolongation of shelf life of food products.
\end{abstract}

\section{Introduction}

Both in the global food market and in the localization of food industry due to quarantine measures, the implementation time for communication network between the food producer and the final consumer increases. This is due to the large distances of the market, or to the limitation of contacts under the quarantine. In this regard, issues of increasing shelf life of food products in different ways are being actively studied in the scientific community. Meanwhile, the emphasis is on eco-friendly technologies that preserve using natural or beneficial substances or microorganisms.

The main trend in the food industry development is application of safe ingredients obtained by microbial synthesis, in particular, enzymes, polysaccharides, amino acids, vitamins and bacteriocins [1]. A special place in the preservation of food products is given to the natural substances or beneficial microorganisms that have a preserving effect, in particular bacteriocins, due to their high antimicrobial potential [2].

\footnotetext{
${ }^{*}$ Corresponding author: a.v.diachkova@urfu.ru
} 
Cleveland et al., Bali et al., Gálvez et al. note that scientists have recently increased their interest in using bacteriocins in food formulations as biopreservatives in food products in order to suppress development of pathogenic microflora [3-5].

Theoretical studies of Ohki et al., Wessels et al. and Moll et al. [6-8] mention nisin as a suitable bacteriocin, which provides an increase in product storage by $50-150 \% \%$, due to the fact that nisin has an impact on gram-positive bacteria, Streptoccus and Lactobacillus, inhibiting their growth. The bactericidal effect of nisin is provided by the destruction of the cytoplasmic membrane of the microbial cell, which leads to the release of intracellular fluid, and, accordingly, the death of organelles and cell lysis. Thus, a nisin molecule is bound by phospholipids of the cell wall and cell peptidoglycans by electrostatic interaction, after which the membrane is compressed and the cell is destroyed [9-11].

Attention to the study of bacteriocins is associated with an increase in the number of pathogenic microorganisms that are resistant to antibiotics. Therefore, at present, studies of new antimicrobial components are very important, allowing them to be used in the development of new generation drugs. The authors Zimina et al., Prosekov et al. [12-13] studied the antibacterial activity of Bacillus safensis, Bacillus endopheticus, Bacillus subtilis strains. Microorganism strains were isolated from vegetables grown in the Krasnodar Krai, namely from Manas onions, big beef tomatoes and Kapia bell peppers. It was found that the minimum inhibitory concentration for the isolated strains of Bacillus safensis was $1.5 * 106$ $\mathrm{cfu} / \mathrm{cm}^{3}$, for Bacillus endopheticus $-1.5^{*} 106 \mathrm{cfu} / \mathrm{cm}^{3}$, for Bacillus subtilis $-1.5 * 108 \mathrm{cfu} / \mathrm{cm}^{3}$. It was proved that the isolated bacteriocins belonged to the lantibiotics class. The most common bacteriocins are the bacteriocins of lactic acid bacteria. To assess the antimicrobial potential of lactic acid bacteriocins and the possibilities for their further use, we determined such indicators as the intensity of bacteriocin production by strains of lactic acid bacteria, the minimum inhibitory concentration of bacteriocins produced by these strains and the antimicrobial activity of the produced bacteriocins. A research of Zimina et al. [14] established that the Lactobacillus paracasei B2430 strain has high antimicrobial activity; it had an inhibitory effect on 12 test cultures: E. coli, Alcaligenes faecalis, candida albicans, Pseudomonas libanensis and Staphylococcus warneri, Erwinia aphidicola bacteria, mycobacterium foliorum, Serratia plymuthica bacteria, Erwinia aphidicola bacteria, bacillus endophyticus, Leuconostoc mesenteroides.

As a result of the research, the practical application of bacteriocins was also found in the food industry in the production of dairy desserts, processed cheeses, yoghurts, and sausages in the Western Europe, USA and Russia.

In this regard, the scientific and practical task is reduced to finding a balance between the widespread use of bacteriocin, but reducing its amount in food formulations. Therefore, it is necessary to develop a way to increase its activity. Then, the high effectiveness of bacteriocin as a preservative will be ensured, probably due to the its low content in the food product.

A well-known solution to this problem is photostimulation, acting with the spectral range of light on a microbial cell, accelerating metabolic processes.

This led to the statement of the objective: to study the impact intensity of blue spectrum light on the Lactobacillus lactis growth.

\section{Materials and methods}

We used a one-day culture of the nisin producer - Lactobacillus lactis for the experiment. Plating in an agar medium [9] and incubation at a temperature of $30 \pm 2{ }^{\circ} \mathrm{C}$ for 48 hours ensured storage and maintenance of the culture. Then we did a monthly plating in skimmed milk. Lactobacillus lactis culture was stored at a temperature of 4 to $6{ }^{\circ} \mathrm{C}$ in the refrigerator. These cultures were divided into two groups: control and experimental. 
Control group samples with Lactobacillus lactis were not treated with light before sowing. Experimental group samples were treated with blue light before sowing, with the "AversSan" biolamp (the scientific and production company "Avers", Moscow) with 20 LEDs builtin with a wavelength of 435-470 $\mathrm{nm}$ and an irradiation intensity of $1800 \mathrm{mcd}$ [9], the processing time was 50-60 minutes.

A one-day culture of Lactobacillus lactis $(100 \mathrm{ml})$ was introduced into the fermentation medium ( $40 \mathrm{~g}$ of skimmed milk powder, $400 \mathrm{ml}$ of whey, proteolytic enzyme, $5 \mathrm{~g}$ of glucose and distilled water up to $1000 \mathrm{ml}$ ). Fermentation was carried out for 24 hours using a laboratory Minipro-Lab fermenter (manufactured by Biotechno) at a $\mathrm{pH}$ of 6.8, its level was maintained by adding a $10 \% \mathrm{NaOH}$ solution. The hydrolysis was carried out for two hours at a temperature of $28-30{ }^{\circ} \mathrm{C}$, the duration of sterilization is 30 minutes. Each group was divided into five samples $(\mathrm{n}=5)$. A nisin solution was prepared on a 0.02 hydrochloric acid solution with a nisin content of 5, 10,20, $40 \mathrm{IU} / \mathrm{ml}$ ). To determine the activity of bacteriocin, $0.1 \mathrm{ml}$ of the standard and test solutions were added to the wells, thermostated (temperature of $55^{\circ} \mathrm{C}$, duration of $16-18$ hours), then the diameter of the growth inhibition zones of the Bacilus coagulans test culture was measured [9].

The titer of Lactobacillus lactis cells was determined by plating on an agar medium consisting of meat peptone broth, yeast autolysate, minerals, enzymatic hydrolyzate of fish meal, bacteriological agar, glucose and sodium chloride. In order to study the activity of Lactobacillus lactis cells, M17 nutrient medium was used (made in Germany, composition: soy peptone, meat peptone, casein peptone, yeast extract, lactose, ascorbic acid, magnesium sulfate, sodium hydroxide solution and distilled water). Lactobacillus lactis culture's resistance to oxidative stress was determined by plating on a nutrient medium with $5 \mathrm{mM}$ hydrogen peroxide in Petri dishes and incubation (temperature of $30-32{ }^{\circ} \mathrm{C}$, duration of 48 hours). The sensitivity was studied by the number of the grown colonies. All reactions and dishes were taken into account after 6, 12 and 24 hours of incubation [9].

All observations for each criterion in each group were checked for uniformity. The coefficient of variation in each group at each moment of measurement ranged from $0.3 \%$ to $11.7 \%$, which corresponds to uniformity in each group.

\section{Results and discussion}

The fermentation properties of the control and experimental samples of Lactobacillus Lactis were determined by measuring the activity of nisin and resistance to oxidative stress. Figure 1 presents data on the activity of nisin during growth; data are presented by the average values in each group.

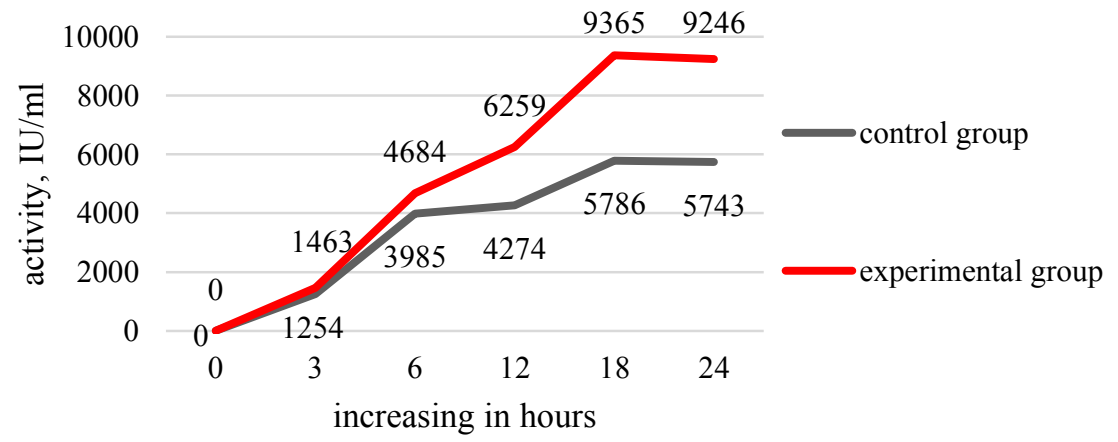

Fig. 1. Dynamics of Lactobacillus lactis activity 
Fig. 1 shows that nisin activity is higher in all experimental group samples than in the control samples. In order to determine the rate of change in nisin activity in samples that were treated with blue light and in the untreated samples, the advance coefficient was calculated. It was calculated as the ratio of the Lactobacillus lactis activity in the experimental group to the corresponding indicators of the control group (Fig. 2).

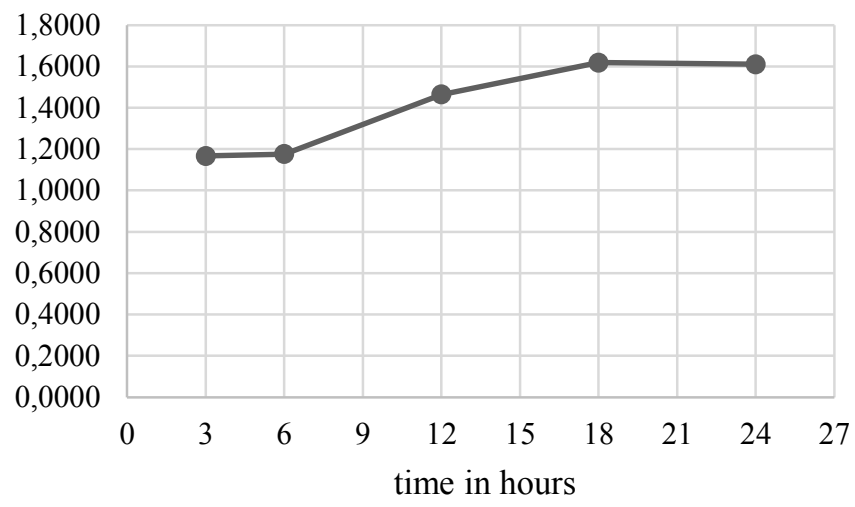

Fig. 2. Advance coefficient: differences in the dynamics of activity of the treated and the untreated bacteriocin

According to Fig. 2 it can be seen that treatment with a blue light lamp provides greater bacteriocin activity throughout all measurements. However, it should be noted that after 18 hours of storage, the activity of the treated bacteriocin slows down. And by 24 hours in the control and experimental groups, the activity of Lactobacillus lactis is reduced.

A two-way analysis of variance revealed the influence of light treatment and time on bacteriocin activity. The value of the F-criterion for the influence of light treatment exceeds the critical F (1204221>2.60597495); therefore, we assumed that the treatment of bacteriocin with blue color affects its activity. The lowest calculated significant difference was 987.41111, which allowed us to conclude that significant differences between the experimental and control groups appear after 12 hours of storage. Until this time, the differences in the actual data of the experimental and control samples are not significant.

The measurement of the titers of cultures of all samples in dynamics has similar conclusions. Fig. 3 presents dynamics of the culture titer, based on the average values of the control and experimental groups.

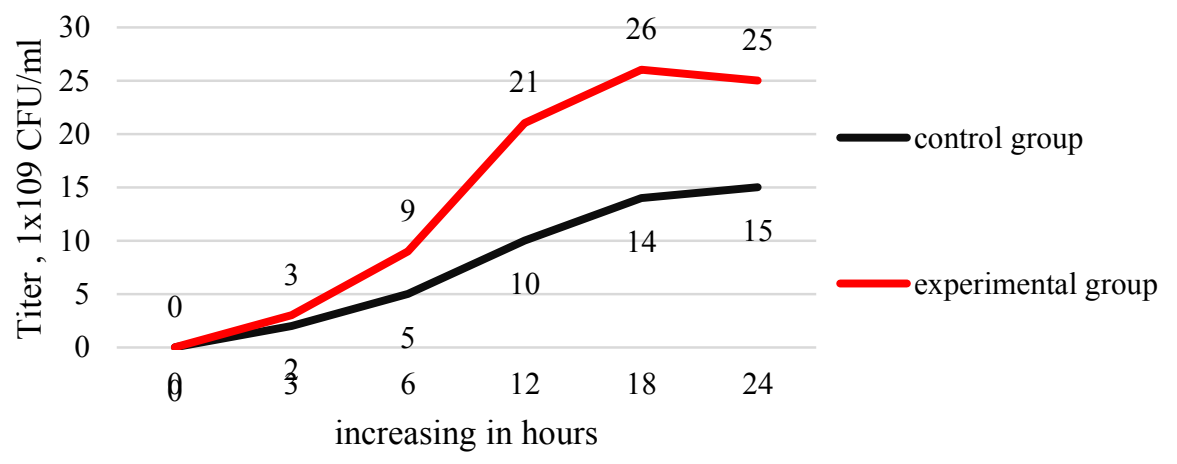

Fig. 3. Increase in the Lactobacillus lactis titer: control and experimental groups 
From Fig. 3 it follows that the titer of the Lactobacillus lactis culture of the experimental group is significantly higher than in the control group. It follows from the results of the study that irradiating the Lactobacillus lactis culture with blue spectrum light $(435-470 \mathrm{~nm})$ with a light flux intensity of 1800 med for 50-60 minutes enhances nisin activity and increases the titer [9]. Meanwhile, a greater increase in the titer is observed in the irradiated samples in the first 18 hours, then the differences between the irradiated and unirradiated samples begin to decrease.

Two-way analysis of variance revealed the influence of light treatment and time on the dynamics of the culture titer $(\mathrm{F}$-criterion $=204.845134$ exceeds $\mathrm{F}$ critical $=2.6059749)$. The lowest calculated significant difference equaled to 0.75885986 and allowed us to conclude that significant differences between the experimental and control groups appear after 3 hours of storage.

We researched the culture's resistance to oxidative stress. A one-day culture of Lactobacillus lactis was placed on an agar medium in Petri dishes (group 1); a culture of Lactobacillus lactis was placed on an agar medium with $5 \mathrm{mM}$ hydrogen peroxide in Petri dishes (group 2); a blue-irradiated culture of Lactobacillus lactis was placed on an agar medium in Petri dishes (group 3); and a blue-irradiated culture of Lactobacillus lactis was placed on an agar medium with $5 \mathrm{mM}$ hydrogen peroxide in Petri dishes (group 4) for this experiment. The sensitivity was investigated by the number of grown colonies, after incubation at a temperature of $30-32{ }^{\circ} \mathrm{C}$ for 48 hours. The reaction was taken into account after 6,12 , and 24 hours of incubation [9]. Fig. 4 presents the effect of hydrogen peroxide on the growth of Lactobacillus lactis in all four groups.

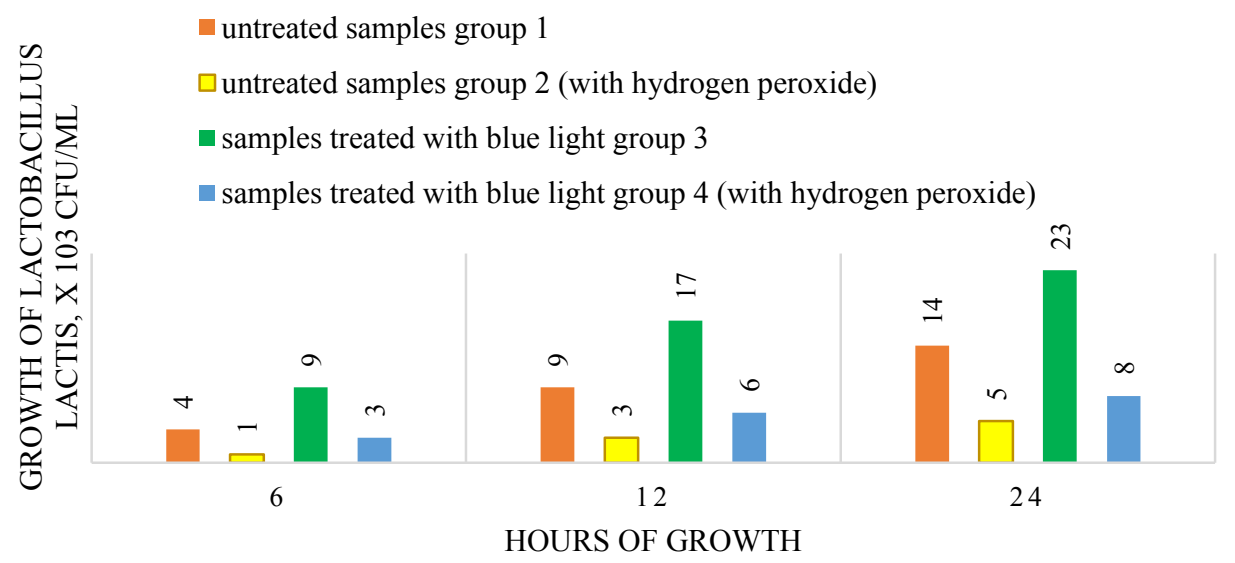

Fig. 4. Impact of hydrogen peroxide on the Lactobacillus lactis growth on the irradiated and unirradiated samples

From the data in Fig. 4 it follows that oxidative stress affects the viability of the culture, both treated with blue light (group 4), and native (group 2). However, the impact intensity to irradiated cultures was lower. This once again confirms that treatment of the Lactobacillus lactis culture with blue light has a positive effect on the growth of microorganisms, but oxidative stress leads to a decrease in the titer [9].

Bacteriocin activity was studied during storage of Lactobacillus lactis culture in skimmed milk for three days at a temperature of 4 to $6{ }^{\circ} \mathrm{C}$ in the refrigerator. 


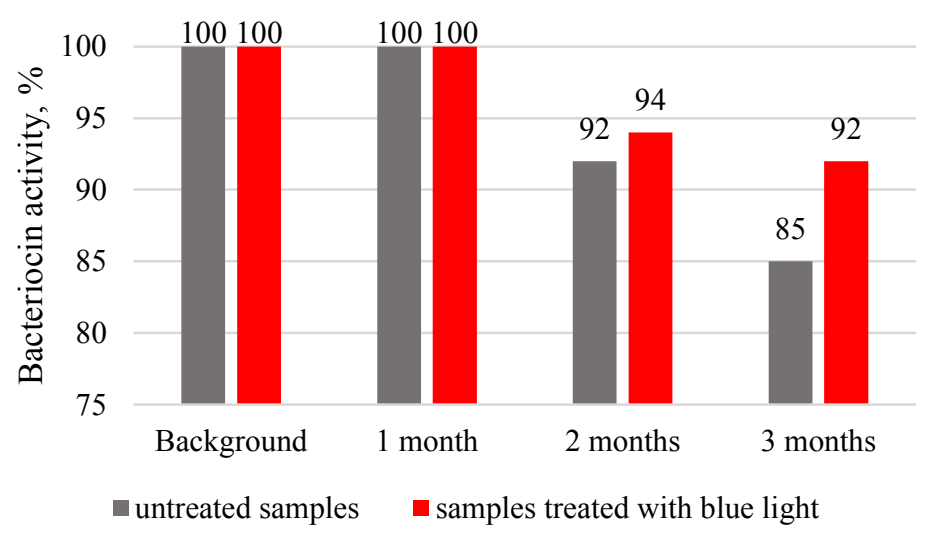

Fig. 5. Bacteriocin activity in the Lactobacillus Lactis samples in skimmed milk, \%

Fig. 5 presents data on the results of a study on storage of the Lactobacillus lactis culture (group 1 - native culture, group 2 - culture samples treated with blue light) in skimmed milk.

The histogram data in Fig. 5 shows that nisin activity is higher during storage in samples of cultures treated with blue spectrum light.

The obtained results indicate the presence of a positive effect of blue spectrum light on the viability of Lactobacillus lactis cells during storage [9].

\section{Conclusion}

Hence, photostimulation of the Lactobacillus Lactis culture with blue light had a positive effect on bacteriocin activity (increased by $60.1 \%$ ), while the titer of the Lactobacillus lactis culture was 2 times higher. Meanwhile, there was a slight decrease in the titer of bacteriocin after 24 hours of incubation. Light treatment of Lactobacillus lactis increases its resistance to oxidative stress and enhances its vitality. The positive effect of light on the viability of bacteriocin activity was proved as a result of studies on the Lactobacillus lactis culture's storage in skimmed milk.

Therefore, photostimulation of the Lactobacillus Lactis culture with blue light has a positive effect on its viability and allows the use of photostimulation for increasing the bacteriocin production.

\section{References}

1. L. Dyshlyuk, O. Babich, D. Belova, A. Prosekov, Journal of Food Process Engineering 40 (2016). DOI: 10.1111/jfpe.12331

2. R. H. Perez, M. T. M. Perez, F. B. Elegado, International Journal of Philippine Science and Technology 8, 61 (2015)

3. J. Cleveland, T. J. Montville, I. F. Nes, M. L. Chikindas, International Journal of Food Microbiology 71, 1 (2001). DOI: 10.1016/S0168-1605(01)00560-8

4. V. Bali, P. S. Panesar, M. B. Bera, J. F. Kennedy, Critical Reviews in Food Science and Nutrition 56, 817 (2014). DOI: 10.1080/10408398.2012.729231

5. A. Gálvez, H. Abriouel, R. L. López, N. B. Omar, International Journal of Food Microbiology 120, 51 (2007). DOI: 10.1016/j.ijfoodmicro.2007.06.001 
6. R. Ohki, K. Tateno, Y. Okada, H. Okajima, K. Asai, Y. Sadaie, M. Murata, T. Aiso, Journal of Bacteriology 185, 51 (2003). DOI: 10.1128/jb.185.1.51-59.2003

7. S. Wessels, B. Jelle, I. Nes, Bacteriocins of the Lactic Acid Bacteria: An Overlooked Benefit for Food Danish Toxicology Centre (Hoersholm, Denmark, 1998)

8. G. N. Moll, W. N. Konings, A. J. Driessen, Antonie van Leeuwenhoek 76, 185 (1999)

9. A. Nogina, Razrabotka tekhnologii biorazlagaemoj plenki dlya uvelicheniya prodolzhitel'nosti hraneniya myasnyh polufabrikatov [Development of biodegradable film technology to increase the shelf life of processed meat] (Kemerovo, 2020)

10. E. Breukink, I. Wiedemann, C. van Kraaij, O. P. Kuipers, H. G. Sahl, B. de Kruijff, Science 286, 2361 (1999). DOI: 10.1126/science.286.5448.2361

11. J. Delves-Broughton, Food Australia 57, 525 (2005)

12. M. I. Zimina, S. A. Sukhih, O. O. Babich, S. Yu. Noskova, A. A. Abrashina, A. Yu. Prosekov, Foods and Raw Materials 4, 92 (2016). DOI: 10.21179/2308-4057-2016-292-100

13. A. Yu. Prosekov, L. S. Dyshlyuk, I. S. Milentyeva, S. A. Sykhikh, O. O. Babich, S. A. Ivanova V. A. Pavsky M. V. Shishin, L. V. Matskova, Progress in Nutrition 19, 67 (2017). DOI: 10.23751/pn.v19i1.5147

14. M. I. Zimina, A. F. Gazieva, J. Pozo-Dengra, S. Yu. Noskova, A. Yu. Prosekov, Foods and Raw Materials 5, 108 (2017). DOI: 10.21179/2308-4057-2017-1-108-117 